\title{
Synthesis and Cytotoxic Activity of Pyranocarbazole Analogues of Ellipticine and Acronycine
}

\author{
Hong Anh Tran-Thi, ${ }^{a}$ Thuan Nguyen-Thi, ${ }^{a}$ Sylvie Michel, ${ }^{a}$ Francois Tillequin, ${ }^{a}$ Michel Koch, ${ }^{a}$ \\ Bruno Pfeiffer, ${ }^{b}$ Alain Pierré, ${ }^{c}$ and Hanh Trinh-VAn-DufaT ${ }^{*} a$ \\ ${ }^{a}$ Laboratoire de Pharmacognosie de l'Université René Descartes, U.M.R./C.N.R.S. $N^{\circ}$ 8638, Faculté des Sciences \\ Pharmaceutiques et Biologiques; 4 Avenue de l'Observatoire, 75006 Paris, France: ${ }^{b}$ Les Laboratoires Servier, and Institut \\ de Recherches Internationales Servier; 1 rue Carle Hébert, 92415 Courbevoie Cedex, France: and ${ }^{c}$ Division Recherche \\ Cancérologie, 125 Chemin de Ronde; 78290 Croissy sur Seine, France. \\ Received November 21, 2003; accepted February 5, 2004
}

Various 2,2,5,11-tetramethyl- and 2,2,5,6,11-pentamethyl-2,6-dihydropyrano[3,2-b]carbazole derivatives were synthesized by condensation of 3-methylbut-2-enal or 3-chloro-3-methylbut-1-yne with an appropriate hydroxycarbazole. These compounds associate the tricyclic system responsible for the intercalating properties of ellipticine related drugs, with the dimethylpyran pharmacophore of acronycine derivatives. The study of the biological properties of the new pyrano[3,2-b]carbazole derivatives was carried out in vitro on L1210 murine leukaemia cell line. The three ( \pm )-cis-diol diesters 15,16 , and 18 were the most active compounds.

Key words ellipticine; acronycine; 2,6-dihydropyrano[3,2-b]carbazole; cytotoxicity

Natural products including a carbazole basic skeleton fused with another heterocyclic system currently receive a strong attention, due to the promising antitumor properties of several of their representatives. ${ }^{1)}$ Of particular interest in this field are the pyrido[4,3- $b$ ]carbazole alkaloids and the indolo[2,3-a]carbazole antibiotics. The first series, illustrated by ellipticine (1) and 9-methoxyellipticine (2), both isolated from Ochrosia species (Apocynaceae) include compounds which act by intercalation within the DNA-double strand, followed by inhibition of topoisomerase II. ${ }^{2)} \mathrm{A}$ derivative of ellipticine, elliptinium (3), has been commercialized for the treatment of metastatic breast cancer. ${ }^{3)}$ Rebeccamycine (4), isolated from Saccharothrix aerocolonigenes, is a typical representative of the second series currently under clinical trials. It induces topoisomerase I mediated DNA-cleavage. ${ }^{4)}$ The antitumor activity of several related indolocarbazoles has been recently discussed. ${ }^{5)}$

The structurally related pyrano[2,3-c]acridone alkaloid acronycine (5) isolated from Achronycia baueri Sснотт. (Rutaceae) was shown to exhibit antitumor properties against a broad spectrum of solid tumor models. ${ }^{6-8)}$ Nevertheless, its moderate potency and low water solubility rapidly appeared as severe drawbacks for a possible introduction into the clinic. A hypothesis of bioactivation of the 1,2-double bond of the chromene ring system of acronycine into the corresponding epoxide ${ }^{9)}$ led to the development of several series of more active structural analogues. The most significant improvements were obtained with diesters of 1,2-dihydroxy1,2-dihydroacronycine ${ }^{10)}$ and, more recently, of 1,2-dihydroxy-1,2-dihydrobenzo[b]acronycine. ${ }^{11)}$ Representatives of this latter series, such as diacetate $\mathbf{6}$, are considered as valuable candidates for clinical studies. ${ }^{12}$ The mechanism of their action implies alkylation of the 2-amino group of DNA guanine residues by the ester leaving group at position 1 of the drug. ${ }^{13,14)}$

We describe here the synthesis and cytotoxic properties of various 2,2,5,11-tetramethyl- and 2,2,5,6,11-pentamethyl2,6-dihydropyrano[3,2-b]carbazole derivatives. Such compounds associate the 1,4-dimethylcarbazole tricyclic system responsible for the intercalating properties of ellipticine related drugs, with the dimethylpyran pharmacophore of acronycine derivatives.

\section{Chemistry}

For the synthesis of 2,2,5,11-tetramethyl-2,6-dihydropyrano[3,2-b]carbazole (7), the readily available 1,4-dimethyl$9 H$-carbazole-3-carbaldehyde (8), previously used as key-intermediate in several ellipticine syntheses, $\left.{ }^{15}\right)$ was chosen as starting material. It was first oxidized by use hydrogen peroxide in acidic medium, into the corresponding 1,4-dimethyl9H-carbazol-3-ol (9), according to the Matsumoto's procedure which has been applied by Langendoen et al. to the synthesis of related hydroxycarbazoles. ${ }^{16)}$ Construction of the fused dimethylpyran ring was ensured by condensation of 9

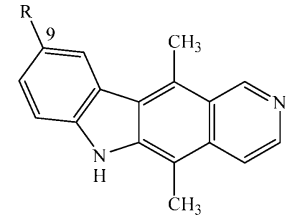

$1 \mathrm{R}=\mathrm{H} \quad$ Ellipticine $2 \mathrm{R}=\mathrm{OCH}_{3}$ 9-Methoxyellipticine

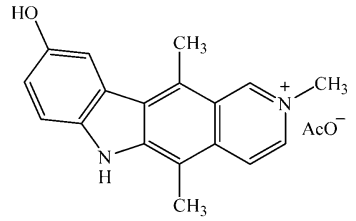

3 Elliptinium

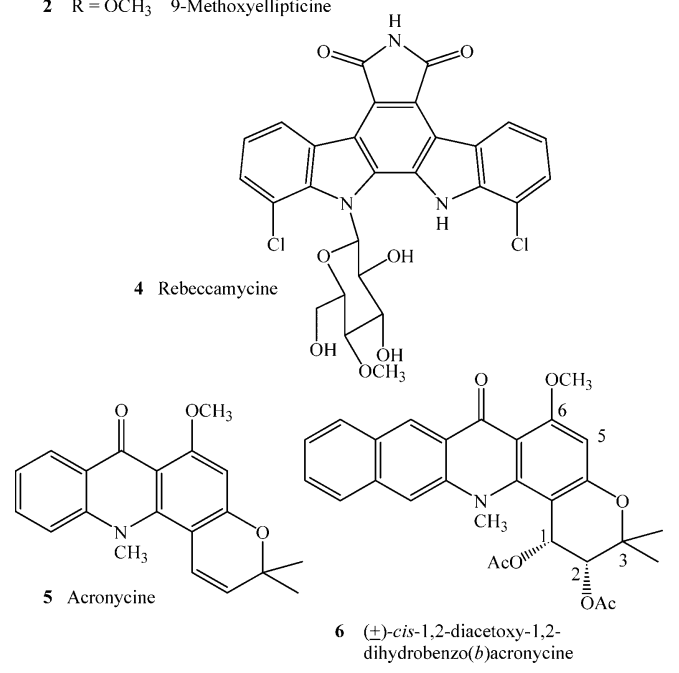


<smiles>[R]c1ccc2[nH]c3c(C)cc(C=O)c(C)c3c2c1</smiles>

$10 \mathrm{R}=\mathrm{OCH}_{3} \quad 69 \%$

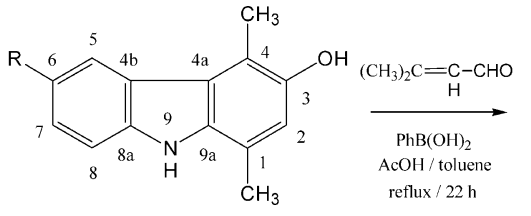

$9 \mathrm{R}=\mathrm{H} \quad 88 \%$

$11 \mathrm{R}=\mathrm{OCH}_{3} \quad 91 \%$

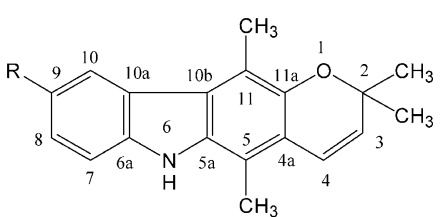

$7 \mathrm{R}=\mathrm{H} \quad 27 \%$

$12 \mathrm{R}=\mathrm{OCH}_{3} \quad 29 \%$

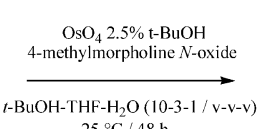

$25{ }^{\circ} \mathrm{C} / 48 \mathrm{~h}$

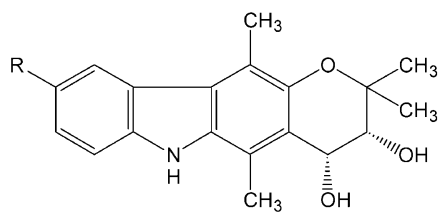

(+)-13 R $=\mathrm{H} \quad 93 \%$

$(+)-14 \quad \mathrm{R}=\mathrm{OCH}_{3} \quad 68 \%$

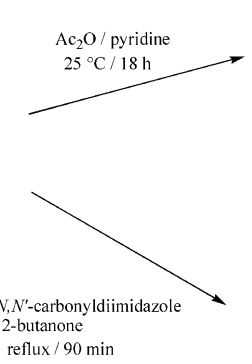

$(+)-17 \mathrm{R}=\mathrm{H}$ $(+)-18 \quad \mathrm{R}=\mathrm{OCH}_{3} \quad 53 \%$<smiles>[R]c1ccc2[nH]c3c(C)c4c(c(C)c3c2c1)OC(C)(C)[C@H](OC(C)=O)[C@H]4OC(C)=O</smiles>

(+)-15 R $=\mathrm{H} \quad 90 \%$ $(+)-16 \quad \mathrm{R}=\mathrm{OCH}_{3} \quad 79 \%$<smiles>[R]c1ccc2[nH]c3c(C)c4c(c(C)c3c2c1)OC(C)(C)[C@H]1OC(=O)O[C@H]41</smiles>

Chart 1<smiles>Cc1c(C=O)cc(C)c2c1c1ccccc1n2C</smiles>

21<smiles>Cc1cc(O)c(C)c2c1c1ccccc1n2C</smiles>

$20 \quad 88 \%$

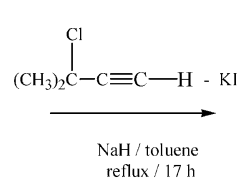

reflux $/ 17 \mathrm{~h}$

Chart 2

with 3-methylbut-2-enal (senecioaldehyde) in the presence of phenylboronic acid as Lewis acid catalyst ${ }^{17)}$ which gave the target compound 7 . The same reaction sequence, applied to 6-methoxy-1,4-dimethyl-9H-carbazole-3-carbaldehyde (10), ${ }^{15)}$ furnished successively 6-methoxy-1,4-dimethyl-9Hcarbazol-3-ol (11) and 9-methoxy-2,2,5,11-tetramethyl-2,6dihydropyrano[3,2-b]carbazole (12) (Chart 1).

Functionalization of the pyran 3,4-double bond of 7 and 12 in order to prepare corresponding $( \pm)$-cis-dihydrodiol diesters was further envisaged, since this pharmacomodulation had previously given outstanding results in terms of antitumor potency in the acronycine series. ${ }^{10)}$

Accordingly, the racemic cis-diols 13 and 14 were conveniently obtained by catalytic osmium tetroxide oxidation of 7 and 12, using $N$-methylmorpholine $N$-oxide to regenerate the oxidizing agent. ${ }^{18)}$ Treatment of diols 13 and 14 with excess acetic anhydride afforded the desired diacetates $\mathbf{1 5}$ and 16, respectively. Reaction of the same diols with $N, N^{\prime}$-carbonydiimidazole in 2-butanone under reflux afforded the corresponding cyclic carbamates $\mathbf{1 7}$ and $\mathbf{1 8}$.

An alternative approach involving the Claisen rearrangement of an intermediate dimethylpropargylic ether was envi- sioned to create the dimethylpyran ring of 2,2,5,6,11-pentamethyl-2,6-dihydropyrano[3,2-b]carbazole (19). ${ }^{19)}$ The starting 1,4,9-trimethyl-9H-carbazol-3-ol (20) was first conveniently prepared by hydrogen peroxide oxidation of the known 1,4,9-trimethyl-9H-carbazole-3-carbaldehyde (21). ${ }^{15}$ Further treatment of $\mathbf{2 0}$ with 3-chloro-3-methylbut-1-yne ${ }^{20)}$ in the presence of sodium hydride in refluxing toluene afforded the desired 19 (Chart 2). Nevertheless, the difficulties encountered in both terms of yield and separation of isomers in the course of the preparation of 21 from 1,4,9-trimethylcarbazole severely limited the practical scope of syntheses using $N$-methycarbazoles as starting materials. In contrast, $N$-methylation of a preformed pyranocarbazole gave excellent results, illustrated by the obtainment in $89 \%$ yield of 9methoxy-2,2,5,6,11-pentamethyl-2,6-dihydropyrano[3,2$b$ ]carbazole (22), when 12 was treated by methyl iodide and sodium hydride in dimethylformamide (Chart 3).

\section{Pharmacology}

The study of the biological properties of the new pyrano[3,2-b]carbazole derivatives was carried out in vitro on L1210 murine leukaemia cell line. The results $\left(\mathrm{IC}_{50}\right)$ are 
<smiles>COc1ccc2[nH]c3c(C)c4c(c(C)c3c2c1)OC(C)(C)C=C4</smiles>

12
$\mathrm{CH}_{3} \mathrm{I}$

$\mathrm{NaH} / \mathrm{DMF}$<smiles>COc1ccc2c(c1)c1c(C)c3c(c(C)c1n2C)C=CC(C)(C)O3</smiles>

$22 \quad 89 \%$

Chart 3

Table 1. Inhibition of L1210 Cell Proliferation

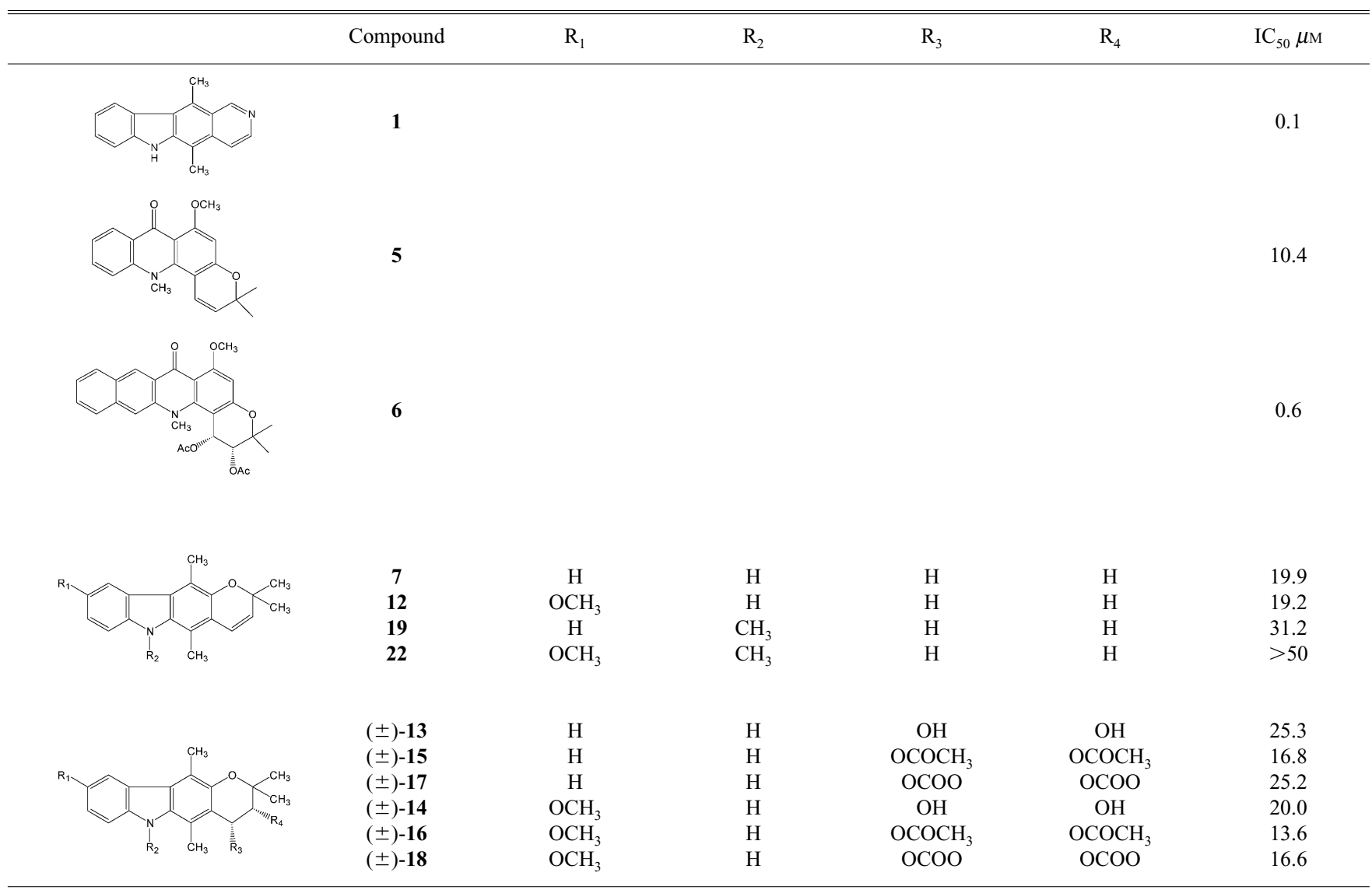

reported in Table 1.

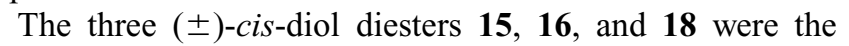
most active compounds.

\section{Results and Discussion}

From a chemical point of view, no synthetic method giving a general entry to the pyrano[3,2-b]carbazole system had been developed up to now. Consequently, only two compounds deriving from this basic skeleton have been described in the literature to our knowledge. Pyrayaquinone-A is the only natural product belonging to this series. It was first isolated from Murraya euchrestifolia HAYATA (Rutaceae) and subsequently synthesized, although in low yield, by palladium-assisted intramolecular ring closure of a suitable chromenylamino-1,4-benzoquinone. ${ }^{21)}$ The other pyrano[3,2$b$ carbazole previously described, isoglycomaurin, was obtained as a minor product in the course of the synthesis of glycomaurin, through Claisen rearrangement of the corresponding dimethylpropargylic ether. ${ }^{22)}$ Therefore, the condensation of a hydroxycarbazole with 3-methylbut-2-enal in presence of a Lewis acid catalyst described here, appears as the first efficient method giving a facile access to the pyrano[3,2- $b]$ carbazole series.

Considering the structure-activity relationships, it appears that in both 2,2,5,11-tetramethyl- and 6-methoxy-2,2,5,11tetramethyl-2,6-dihydropyrano[3,2-b]carbazole series, functionalization of the pyran double bond to give ( \pm )-cis-dihydro-diol diesters results in an increase of the cytotoxic activity. Nevertheless, none of these esters exhibit an antiproliferative activity within the same range of magnitude as the corresponding acronycine derivatives.

In contrast, $N$-methylation reduces significantly the biological activity in both chemical series. Indeed, 19 only exhibits a marginal activity, whereas $\mathbf{2 2}$ was devoid significant cytotoxic activity.

The effect of the substitution by a methoxy group at position 9 seems to be more discrete.

However, methoxy compounds appear, as a whole, slightly more cytotoxic than their unsubstituted counterparts. 


\section{Experimental}

The melting points were determined on a Leica VM apparatus and are not corrected. IR spectra $\left(v_{\max }\right.$ in $\left.\mathrm{cm}^{-1}\right)$ with $\mathrm{KBr}$ pellets were obtained on a Nicolet FT-IR instrument. UV spectra $\left(\lambda_{\max }\right.$ in $\left.\mathrm{nm}\right)$ were determined in $>99.5 \%(\mathrm{v} / \mathrm{v}) \mathrm{EtOH}$ on a Beckman $\mathrm{DU}^{\circledR} 600$ spectrophotometer. ${ }^{1} \mathrm{H}-\mathrm{NMR}$ ( $\delta$ in ppm, $J$ in $\mathrm{Hz}$ ) and ${ }^{13} \mathrm{C}-\mathrm{NMR}$ spectra were recorded at $400 \mathrm{MHz}$ and $100 \mathrm{MHz}$ respectively, using a Bruker Avance 400 spectrometer. When necessary, the signals were unambiguously assigned by $2 \mathrm{D}$ NMR techniques: COSY, NOESY, HMQC, and HMBC. These experiments were performed using standard Bruker microprograms. Mass spectra were recorded with a Nermag R-10-10C spectrometer using chemical ionization technique (reagent gas: $\mathrm{NH}_{3}$ ) (CI-MS) and with a ZQ 2000 Waters using a Zspray (ESI-MS). Column chromatographies were conducted using silica gel 60 Merck $(35-70 \mu \mathrm{m})$ with an overpressure of 200 mbars.

1,4-Dimethyl-9H-carbazol-3-ol (9) To an ice-cooled solution of 1,4-dimethyl- $9 H$-carbazole-3-carbaldehyde ${ }^{15)}(7.8 \mathrm{~g}, 35.0 \mathrm{mmol})$ in methanol $(400 \mathrm{ml})$ were added $33 \%$ aqueous hydrogen peroxide $(5 \mathrm{ml})$ and $95 \%$ sulfuric acid $(2.5 \mathrm{ml})$. After stirring for $15 \mathrm{~min}$ at $0{ }^{\circ} \mathrm{C}$, the reaction mixture was diluted with a mixture of crushed ice and water and neutralized with aqueous $1 \mathrm{~m} \mathrm{NaOH}$ to give a whitish precipitate which was filtered off and washed with water. Crystallization from $\mathrm{MeOH}$ gave 1,4-dimethyl- $9 H$-carbazol-3-ol as whitish needles $(6.5 \mathrm{~g}, 88 \%)$, mp 246-247 ${ }^{\circ} \mathrm{C} .{ }^{1} \mathrm{H}-\mathrm{NMR}$ $\left(\right.$ DMSO- $\left.d_{6}\right) \delta: 2.45\left(3 \mathrm{H}, \mathrm{s}, \mathrm{CH}_{3}-1\right), 2.60\left(3 \mathrm{H}, \mathrm{s}, \mathrm{CH}_{3}-4\right), 6.77(1 \mathrm{H}, \mathrm{s}, \mathrm{C} 2-\mathrm{H})$, $7.07(1 \mathrm{H}, \mathrm{t}, J=8 \mathrm{~Hz}, \mathrm{C} 6-\mathrm{H}), 7.30(1 \mathrm{H}, \mathrm{t}, J=8 \mathrm{~Hz}, \mathrm{C} 7-\mathrm{H}), 7.44(1 \mathrm{H}, \mathrm{d}$, $J=8 \mathrm{~Hz}, \mathrm{C} 8-\mathrm{H}), 8.08(1 \mathrm{H}, \mathrm{d}, J=8 \mathrm{~Hz}, \mathrm{C} 5-\mathrm{H}), 8.65\left(1 \mathrm{H}, \mathrm{D}_{2} \mathrm{O}\right.$ exch, $\left.\mathrm{s}, \mathrm{OH}\right)$, $10.80\left(1 \mathrm{H}, \mathrm{D}_{2} \mathrm{O}\right.$ exch, s, NH). ${ }^{13} \mathrm{C}-\mathrm{NMR}\left(\mathrm{DMSO}-d_{6}\right) \delta: 13.7\left(\mathrm{CH}_{3}-4\right), 18.1$ $\left(\mathrm{CH}_{3}-1\right), 112.0$ (C-8), 115.1 (C-4a), 116.8 (C-2), 118.3 (C-4), 119.0 (C-6), 122.8 (C-1), 123.3 (C-5), 124.9 (C-4b), 125.6 (C-7), 134.4 (C-9a), 141.7 (C8a), 148.7 (C-3). IR $v_{\max } \mathrm{cm}^{-1}: 3493,3319,3056,2959,2933,2917,1457$, 1306, 1090, 756, 735. UV $\lambda_{\text {max }} \mathrm{nm}(\log \varepsilon): 222$ (4.38), 238 (4.45), 252.5 (4.23), 267.5 (4.23), 295 (4.07). CI-MS m/z: 212 ([MH] $\left.]^{+}\right)$. Anal. Calcd for $\mathrm{C}_{14} \mathrm{H}_{13} \mathrm{NO}$ : C, 79.59; H, 6.20; N, 6.63. Found: C, 79.31; H, 6.18; N, 6.61.

2,2,5,11-Tetramethyl-2,6-dihydropyrano $[3,2-b]$ carbazole (7) A solution of $9(5.0 \mathrm{~g}, 23.7 \mathrm{mmol})$, phenylboronic acid $(4.3 \mathrm{~g}, 35.5 \mathrm{mmol}), 3-$ methylbut-2-enal $(3.0 \mathrm{~g}, 35.5 \mathrm{mmol})$, glacial acetic acid $(90 \mathrm{ml})$ in anhydrous toluene $(300 \mathrm{ml})$ was refluxed for $22 \mathrm{~h}$ under $\mathrm{N}_{2}$ in an apparatus fitted with a Dean-Stark trap. After cooling, the mixture was concentrated in vacuo and the residue was extracted with $\mathrm{CH}_{2} \mathrm{Cl}_{2}(4 \times 100 \mathrm{ml})$. The combined organic phase was washed successively with $\mathrm{H}_{2} \mathrm{O}(150 \mathrm{ml}), 5 \% \mathrm{NaHCO}_{3}$ solution $(200 \mathrm{ml})$, and brine $(150 \mathrm{ml})$, dried over $\mathrm{Na}_{2} \mathrm{SO}_{4}$, filtered, and the solvent was evaporated under reduced pressure. Column chromatography of the crude product on silica gel $\left(\mathrm{CH}_{2} \mathrm{Cl}_{2} /\right.$ cyclohexane: $\left.7 / 3, \mathrm{v} / \mathrm{v}\right)$ gave 7 as a pale yellow crystalline product $(1.76 \mathrm{~g}, 27 \%), \mathrm{mp} 139-140{ }^{\circ} \mathrm{C} .{ }^{1} \mathrm{H}-\mathrm{NMR}\left(\mathrm{CDCl}_{3}\right) \delta$ : $1.45\left(3 \mathrm{H}, \mathrm{s}, \mathrm{CH}_{3}-2\right), 1.48\left(3 \mathrm{H}, \mathrm{s}, \mathrm{CH}_{3}-2\right), 2.50\left(3 \mathrm{H}, \mathrm{s}, \mathrm{CH}_{3}-5\right), 2.75(3 \mathrm{H}, \mathrm{s}$, $\left.\mathrm{CH}_{3}-11\right), 5.81(1 \mathrm{H}, \mathrm{d}, J=10 \mathrm{~Hz}, \mathrm{C} 3-\mathrm{H}), 6.74(1 \mathrm{H}, \mathrm{d}, J=10 \mathrm{~Hz}, \mathrm{C} 4-\mathrm{H}), 7.18$ $(1 \mathrm{H}$, ddd, $J=8,7,1.5 \mathrm{~Hz}, \mathrm{C} 9-\mathrm{H}), 7.37(1 \mathrm{H}, \mathrm{td}, J=7,1 \mathrm{~Hz}, \mathrm{C} 8-\mathrm{H}), 7.43(1 \mathrm{H}$, dd, $J=7,1.5 \mathrm{~Hz}, \mathrm{C} 7-\mathrm{H}), 7.78\left(1 \mathrm{H}, \mathrm{D}_{2} \mathrm{O}\right.$ exch, s, NH), $8.17(1 \mathrm{H}, \mathrm{dd}, J=8$, $1 \mathrm{~Hz}, \mathrm{C} 10-\mathrm{H}) .{ }^{13} \mathrm{C}-\mathrm{NMR}\left(\mathrm{CDCl}_{3}\right) \delta: 12.0\left(\mathrm{CH}_{3}-5\right), 12.2\left(\mathrm{CH}_{3}-11\right), 27.2(2 \mathrm{C}$, $\left.\left(\mathrm{CH}_{3}\right)_{2}-2\right), 74.5(\mathrm{C}-2), 110.4(\mathrm{C}-7), 112.0(\mathrm{C}-4 \mathrm{a}), 117.2(\mathrm{C}-10 \mathrm{~b}), 118.8(\mathrm{C}-$ 5), 118.9 (C-9), 120.3 (C-10), 121.7 (C-11), 122.6 (C-4), 124.7 (C-10a), 124.9 (C-8), 131.8 (C-3), 133.8 (C-5a), 140.2 (C-6a), 144.2 (C-11a). IR $v_{\max }$ $\mathrm{cm}^{-1}: 3412,3050,2973,2915,1301,1147,1135,746,725 . \mathrm{UV} \lambda_{\max } \mathrm{nm}$ $(\log \varepsilon): 258$ (4.58), 313 (4.34), 325 (4.44). CI-MS m/z: $278\left([\mathrm{MH}]^{+}\right)$. Anal. Calcd for $\mathrm{C}_{19} \mathrm{H}_{19}$ NO: C, 82.28; H, 6.90; N, 5.05. Found: C, 82.28; H, 6.88; N, 5.03 .

6-Methoxy-1,4-dimethyl-9H-carbazol-3-ol (11) Oxidation of 6methoxy-1,4-dimethyl-9H-carbazole-3-carbaldehyde $\left.\quad(\mathbf{1 0})^{15}\right) \quad(4.0 \mathrm{~g}, \quad 15.8$ $\mathrm{mmol}$ ) under conditions identical with those described for the preparation of 9, afforded 11 as pale green yellow needles $(3.5 \mathrm{~g}, 91 \%), \mathrm{mp} 185-186^{\circ} \mathrm{C}$. ${ }^{1} \mathrm{H}-\mathrm{NMR}\left(\mathrm{DMSO}-d_{6}\right) \delta: 2.50\left(3 \mathrm{H}, \mathrm{s}, \mathrm{CH}_{3}-1\right), 2.70\left(3 \mathrm{H}, \mathrm{s}, \mathrm{CH}_{3}-4\right), 3.90(3 \mathrm{H}$, $\left.\mathrm{s}, \mathrm{OCH}_{3}\right), 6.85(1 \mathrm{H}, \mathrm{s}, \mathrm{C} 2-\mathrm{H}), 7.04(1 \mathrm{H}, \mathrm{dd}, J=8,1 \mathrm{~Hz}, \mathrm{C} 7-\mathrm{H}), 7.43(1 \mathrm{H}, \mathrm{d}$, $J=8 \mathrm{~Hz}, \mathrm{C} 8-\mathrm{H}), 7.67(1 \mathrm{H}, \mathrm{d}, J=1 \mathrm{~Hz}, \mathrm{C} 5-\mathrm{H}), 8.60\left(1 \mathrm{H}, \mathrm{D}_{2} \mathrm{O}\right.$ exch, s, OH), $10.68\left(1 \mathrm{H}, \mathrm{D}_{2} \mathrm{O}\right.$ exch, s, NH). ${ }^{13} \mathrm{C}-\mathrm{NMR}\left(\mathrm{CDCl}_{3}-\mathrm{CD}_{3} \mathrm{OD}\right) \delta: 12.1\left(\mathrm{CH}_{3}-4\right)$, $16.4\left(\underline{\mathrm{CH}}_{3}-1\right), 56.2\left(\mathrm{OCH}_{3}\right), 106.4(\mathrm{C}-5), 110.9(\mathrm{C}-8), 113.5$ (C-7), 115.1 (C4a), 115.7 (C-2), 117.3 (C-4), 122.3 (C-1), 124.9 (C-4b), 134.6 (C-9a), 135.5 (C-8a), 146.6 (C-3), 153.0 (C-6). IR $v_{\max } \mathrm{cm}^{-1}: 3452,3421,3348$, 2967, 2916, 1482, 1215, 1091, 1020. UV $\lambda_{\max } \mathrm{nm}(\log \varepsilon): 231$ (4.47), 258 (3.05), 271.5 (2.84), 305.5 (3.18), 357 (2.57). CI-MS m/z: $242\left([\mathrm{MH}]^{+}\right)$. Anal. Calcd for $\mathrm{C}_{15} \mathrm{H}_{15} \mathrm{NO}_{2}: \mathrm{C}, 74.67 ; \mathrm{H}, 6.27 ; \mathrm{N}, 5.81$. Found: C, 74.48; H, $6.26 ; \mathrm{N}, 5.79$.

9-Methoxy-2,2,5,11-tetramethyl-2,6-dihydropyrano[3,2-b]carbazole (12) Synthesis of $\mathbf{1 2}$ from $\mathbf{1 1}(3.46 \mathrm{~g}, 14.4 \mathrm{mmol})$ carried out under the same conditions as those applied for the preparation of compound 7, afforded 12 as a pale yellow amorphous product $(1.27 \mathrm{~g}, 29 \%) .{ }^{1} \mathrm{H}-\mathrm{NMR}$
$\left(\mathrm{CDCl}_{3}\right) \delta: 1.48\left(6 \mathrm{H}, \mathrm{s},\left(\mathrm{CH}_{3}\right)_{2}-2\right), 2.45\left(3 \mathrm{H}, \mathrm{s}, \mathrm{CH}_{3}-5\right), 2.72\left(3 \mathrm{H}, \mathrm{s}, \mathrm{CH}_{3}-\right.$ 11), $3.95\left(3 \mathrm{H}, \mathrm{s}, \mathrm{OCH}_{3}\right), 5.81(1 \mathrm{H}, \mathrm{d}, J=10 \mathrm{~Hz}, \mathrm{C} 3-\mathrm{H}), 6.73(1 \mathrm{H}, \mathrm{d}$, $J=10 \mathrm{~Hz}, \mathrm{C} 4-\mathrm{H}), 7.03(1 \mathrm{H}, \mathrm{dd}, J=9,2.5 \mathrm{~Hz}, \mathrm{C} 8-\mathrm{H}), 7.32(1 \mathrm{H}, \mathrm{d}, J=9 \mathrm{~Hz}$, $\mathrm{C} 7-\mathrm{H}), 7.64\left(1 \mathrm{H}, \mathrm{D}_{2} \mathrm{O}\right.$ exch, s, NH), $7.68(1 \mathrm{H}, \mathrm{d}, J=2.5 \mathrm{~Hz}, \mathrm{C} 10-\mathrm{H}) .{ }^{13} \mathrm{C}-$ NMR $\left(\mathrm{CDCl}_{3}\right) \delta: 12.0\left(\underline{\mathrm{CH}}_{3}-11\right), 12.1\left(\underline{\mathrm{CH}}_{3}-5\right), 27.2\left(2 \mathrm{C},\left(\mathrm{CH}_{3}\right)_{2}-2\right), 56.2$ $\left(\mathrm{OCH}_{3}\right), 74.4$ (C-2), 106.3 (C-10), 110.9 (C-7), 112.1 (C-4a), 113.6 (C-8), 117.0 (C-10b), 118.8 (C-5), 120.3 (C-4), 121.8 (C-11), 125.3 (C-10a), 131.9 (C-3), 134.9 (C-5a), 135.5 (C-6a), 144.0 (C-11a), 153.4 (C-9). IR $v_{\max } \mathrm{cm}^{-1}$ : $3431,3356,3045,2961,2922,1482,1314,1214,1135$. UV $\lambda_{\max } \mathrm{nm}(\log \varepsilon)$ : 210 (4.15), 257.5 (4.52), 282 (3.84), 321.5 (4.31), 334 (4.44). CI-MS m/z: $307\left([\mathrm{MH}]^{+}\right)$. Anal. Calcd for $\mathrm{C}_{20} \mathrm{H}_{21} \mathrm{NO}_{2}: \mathrm{C}, 78.15 ; \mathrm{H}, 6.89 ; \mathrm{N}, 4.56$. Found: C, 78.02; H, 6.88; N, 4.57.

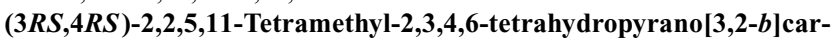
bazole-3,4-diol (13) Compound $7(135 \mathrm{mg}, 0.49 \mathrm{mmol})$ was added to a solution of osmium tetroxide (2.5\% in 2-methyl-2-propanol) $(0.31 \mathrm{ml})$ and $N$ methylmorpholine $N$-oxide dihydrate $(65 \mathrm{mg}, 0.54 \mathrm{mmol})$ in a mixture of $t$ $\mathrm{BuOH} / \mathrm{THF} / \mathrm{H}_{2} \mathrm{O}: 10 / 3 / 1, \mathrm{v} / \mathrm{v} / \mathrm{v}(3 \mathrm{ml})$. The reaction mixture was stirred at $25^{\circ} \mathrm{C}$ for $48 \mathrm{~h}$. After addition of aqueous saturated $\mathrm{NaHSO}_{3}$ and stirring for $1 \mathrm{~h}$, the mixture was extracted with $\mathrm{CH}_{2} \mathrm{Cl}_{2}(3 \times 50 \mathrm{ml})$. The combined organic layers were dried over $\mathrm{Na}_{2} \mathrm{SO}_{4}$, filtered, and evaporated under reduced pressure. Column chromatography of the crude product on silica gel $\left(\mathrm{CH}_{2} \mathrm{Cl}_{2} / \mathrm{MeOH}\right.$ : $\left.99 / 1, \mathrm{v} / \mathrm{v}\right)$ gave $\mathbf{1 3}$ as a whitish amorphous product (141 mg, 93\%). ${ }^{1} \mathrm{H}-\mathrm{NMR}\left(\mathrm{CD}_{3} \mathrm{OD}\right) \delta: 1.38\left(3 \mathrm{H}, \mathrm{s}, \mathrm{CH}_{3}-2\right), 1.45(3 \mathrm{H}, \mathrm{s}$, $\left.\mathrm{CH}_{3}-2\right), 2.58\left(3 \mathrm{H}, \mathrm{s}, \mathrm{CH}_{3}-5\right), 2.65\left(3 \mathrm{H}, \mathrm{s}, \mathrm{CH}_{3}-11\right), 3.75(1 \mathrm{H}, \mathrm{d}, J=5 \mathrm{~Hz}, \mathrm{C} 3-$ $\mathrm{H}), 4.97(1 \mathrm{H}, \mathrm{d}, J=5 \mathrm{~Hz}, \mathrm{C} 4-\mathrm{H}), 7.06(1 \mathrm{H}, \mathrm{td}, J=8,1 \mathrm{~Hz}, \mathrm{C} 9-\mathrm{H}), 7.30(1 \mathrm{H}$, td, $J=8,1 \mathrm{~Hz}, \mathrm{C} 8-\mathrm{H}), 7.42(1 \mathrm{H}, \mathrm{dd}, J=8,1 \mathrm{~Hz}, \mathrm{C} 7-\mathrm{H}), 8.10(1 \mathrm{H}, \mathrm{dd}, J=8$, $1 \mathrm{~Hz}, \mathrm{C} 10-\mathrm{H}) .{ }^{13} \mathrm{C}-\mathrm{NMR}\left(\mathrm{CD}_{3} \mathrm{OD}\right) \delta: 12.7\left(\underline{\mathrm{CH}}_{3}-5\right), 13.4\left(\underline{\mathrm{CH}}_{3}-11\right), 21.4$ $\left(\underline{\mathrm{CH}}_{3}-2\right), 27.2\left(\mathrm{CH}_{3}-2\right), 66.1(\mathrm{C}-4), 74.6(\mathrm{C}-3), 77.0(\mathrm{C}-2), 111.6(2 \mathrm{C}, \mathrm{C}-7$ and C-4a), 117.5 (C-10b), 118.6 (C-5), 119.0 (C-9), 121.3 (C-11), 123.5 (C10), 125.3 (C-10a), 125.9 (C-8), 135.8 (C-5a), 142.6 (C-6a), 144.8 (C-11a). IR $v_{\max } \mathrm{cm}^{-1}: 3523,3450,3362,3282,3050,2971,2918,1504,1458,1392$, $1301,1149,1102,1007,746$. UV $\lambda_{\max } \mathrm{nm}(\log \varepsilon): 224$ (4.42), 240 (4.57), 257.5 (4.31), 271 (4.09), 290 (4.08), 299 (4.32). CI-MS $m / z: 312\left([\mathrm{MH}]^{+}\right)$. Anal. Calcd for $\mathrm{C}_{19} \mathrm{H}_{21} \mathrm{NO}_{3}: \mathrm{C}, 73.29 ; \mathrm{H}, 6.80 ; \mathrm{N}, 4.50$. Found: C, 73.12; H, $6.82 ; \mathrm{N}, 4.51$.

$(3 R S, 4 R S)$-9-Methoxy-2,2,5,11-tetramethyl-2,3,4,6-tetrahydropyrano[3,2-b]carbazole-3,4-diol (14) Oxidation of $12(50 \mathrm{mg}, 0.16 \mathrm{mmol})$ according to the same procedure as that described for the preparation of $\mathbf{1 3}$, afforded diol 14 as a whitish amorphous product $(38 \mathrm{mg}, 68 \%) .{ }^{1} \mathrm{H}-\mathrm{NMR}$ $\left(\right.$ DMSO- $\left.d_{6}\right) \delta: 1.30\left(3 \mathrm{H}, \mathrm{s}, \mathrm{CH}_{3}-2\right), 1.41\left(3 \mathrm{H}, \mathrm{s}, \mathrm{CH}_{3}-2\right), 2.52\left(3 \mathrm{H}, \mathrm{s}, \mathrm{CH}_{3}-\right.$ 5), $2.60\left(3 \mathrm{H}, \mathrm{s}, \mathrm{CH}_{3}-11\right), 3.65(1 \mathrm{H}, \mathrm{dd}, J=7,5 \mathrm{~Hz}, \mathrm{C} 3-\mathrm{H}), 3.83(3 \mathrm{H}, \mathrm{s}$, $\left.\mathrm{OCH}_{3}\right), 4.78\left(1 \mathrm{H}, \mathrm{D}_{2} \mathrm{O}\right.$ exch, d, $\left.J=5 \mathrm{~Hz}, \mathrm{C} 4-\mathrm{OH}\right), 4.85(1 \mathrm{H}, \mathrm{t}, J=5 \mathrm{~Hz}, \mathrm{C} 4-$ H), $5.00\left(1 \mathrm{H}, \mathrm{D}_{2} \mathrm{O}\right.$ exch, d, $\left.J=5 \mathrm{~Hz}, \mathrm{C} 3-\mathrm{OH}\right), 7.01(1 \mathrm{H}, \mathrm{dd}, J=9,2 \mathrm{~Hz}, \mathrm{C} 8-$ H), $7.37(1 \mathrm{H}, \mathrm{d}, J=9 \mathrm{~Hz}, \mathrm{C} 7-\mathrm{H}), 7.59(1 \mathrm{H}, \mathrm{d}, J=2 \mathrm{~Hz}, \mathrm{C} 10-\mathrm{H}), 10.60(1 \mathrm{H}$, $\mathrm{D}_{2} \mathrm{O}$ exch, s, NH). ${ }^{13} \mathrm{C}-\mathrm{NMR}$ (DMSO- $\left.d_{6}\right) \delta: 12.8\left(\mathrm{CH}_{3}-11\right), 13.7\left(\underline{\mathrm{CH}}_{3}-5\right)$, $21.5\left(\mathrm{CH}_{3}-2\right), 27.8\left(\mathrm{CH}_{3}-2\right), 56.2\left(\mathrm{OCH}_{3}\right), 64.5(\mathrm{C}-4), 73.2(\mathrm{C}-3), 76.3(\mathrm{C}-$ 2), 106.1 (C-10), 111.8 (C-7), 114.2 (C-8), 115.4 (C-10b), 118.2 (C-4a), 121.7 (C-11), 121.9 (C-5), 124.2 (C-10a), 135.5 (C-5a), 136.4 (C-6a), 143.4 (C-11a), 152.9 (C-9). IR $v_{\max } \mathrm{cm}^{-1}: 3557,3431,3271,2990,2916,2870$, $1480,1218,1145,1095$. UV $\lambda_{\max } \mathrm{nm}(\log \varepsilon): 238(4.52), 250(4.35), 260.5$ (4.08), 274.5 (3.68), 299 (4.09), 308.5 (4.34). ESI-MS $m / z: 364\left([\mathrm{M}+\mathrm{Na}]^{+}\right)$. Anal. Calcd for $\mathrm{C}_{20} \mathrm{H}_{23} \mathrm{NO}_{4}$ : C, 70.36; $\mathrm{H}, 6.79 ; \mathrm{N}, 4.10$. Found: $\mathrm{C}, 70.35 ; \mathrm{H}$, $6.80 ; \mathrm{N}, 4.11$.

$(3 R S, 4 R S)-2,2,5,11-T e t r a m e t h y l-2,3,4,6$-tetrahydropyrano[3,2-b]carbazole-3,4-diyle Diacetate (15) Diol 13 (305 mg, $0.98 \mathrm{mmol})$ was acetylated with acetic anhydride in anhydrous pyridine under $\operatorname{Ar}$ at $25^{\circ} \mathrm{C}$ for $18 \mathrm{~h}$. The mixture was evaporated under reduced pressure and the residue gave $\mathbf{1 5}$ as a pale yellow amorphous product $(349 \mathrm{mg}, 90 \%) .{ }^{1} \mathrm{H}-\mathrm{NMR}$ (DMSO- $d_{6}$ ) $\delta: 1.36\left(3 \mathrm{H}, \mathrm{s}, \mathrm{CH}_{3}-2\right), 1.37\left(3 \mathrm{H}, \mathrm{s}, \mathrm{CH}_{3}-2\right), 2.05\left(3 \mathrm{H}, \mathrm{s}, \mathrm{OCOCH}_{3}\right) 2.07$ $\left(3 \mathrm{H}, \mathrm{s}, \mathrm{OCOCH}_{3}\right), 2.32\left(3 \mathrm{H}, \mathrm{s}, \mathrm{CH}_{3}-5\right), 2.63\left(3 \mathrm{H}, \mathrm{s}, \mathrm{CH}_{3}-11\right), 5.17(1 \mathrm{H}, \mathrm{d}$, $J=5 \mathrm{~Hz}, \mathrm{C} 3-\mathrm{H}), 6.33(1 \mathrm{H}, \mathrm{d}, J=5 \mathrm{~Hz}, \mathrm{C} 4-\mathrm{H}), 7.12(1 \mathrm{H}, \mathrm{t}, J=8 \mathrm{~Hz}, \mathrm{C} 9-\mathrm{H})$, $7.37(1 \mathrm{H}, \mathrm{t}, J=8 \mathrm{~Hz}, \mathrm{C} 8-\mathrm{H}), 7.48(1 \mathrm{H}, \mathrm{d}, J=8 \mathrm{~Hz}, \mathrm{C} 7-\mathrm{H}), 8.12(1 \mathrm{H}, \mathrm{d}$, $J=8 \mathrm{~Hz}, \mathrm{C} 10-\mathrm{H}), 11.00\left(1 \mathrm{H}, \mathrm{D}_{2} \mathrm{O}\right.$ exch, s, NH). ${ }^{13} \mathrm{C}-\mathrm{NMR}$ (DMSO- $\left.d_{6}\right) \delta$ : $12.8\left(\underline{\mathrm{CH}}_{3}-11\right), 13.4\left(\mathrm{CH}_{3}-5\right), 20.9\left(\mathrm{OCO} \mathrm{CH}_{3}\right), 21.1\left(\mathrm{OCO} \mathrm{CH}_{3}\right), 22.1\left(\underline{\mathrm{CH}}_{3}-\right.$ 2), $26.4\left(\mathrm{CH}_{3}-2\right), 64.6$ (C-4), 72.2 (C-3), 74.7 (C-2), 111.5 (C-7), 115.9 (C4a), 116.7 (C-10a), 117.6 (C-5), 118.8 (C-9), 122.8 (C-11), 123.1 (C-10), 123.5 (C-10a), 126.0 (C-8), 134.7 (C-5a), 141.6 (C-6a), 144.0 (C-11a), $170.2\left(\mathrm{COCH}_{3}\right), 170,6\left(\mathrm{COCH}_{3}\right)$. IR $v_{\max } \mathrm{cm}^{-1}: 3406,3049,2990,2940$, $1747,1728,1369,1242,1016,741$. UV $\lambda_{\max } \mathrm{nm}(\log \varepsilon): 240(4.28), 247.5$ (4.22), 258.5 (3.99), 270 (3.71), 290.5 (3.73), 299.5 (4.05). ESI-MS $m / z$ : $418\left([\mathrm{M}+\mathrm{Na}]^{+}\right), 336\left([\mathrm{M}-\mathrm{OAc}]^{+}\right)$. Anal. Calcd for $\mathrm{C}_{23} \mathrm{H}_{25} \mathrm{NO}_{5}: \mathrm{C}, 69.86$; H, 6.37; N, 3.54. Found: C, 69.90; H, 6.40; N, 4.53.

$(3 R S, 4 R S)$-9-Methoxy-2,2,5,11-tetramethyl-2,3,4,6-tetrahydropyrano[3,2-b]carbazole-3,4-diyle Diacetate (16) Diol 14 (80 mg, 0.23 
mmol) was acetylated according to the same procedure as that used for the preparation of compound $\mathbf{1 5}$, to afford the diacetate $\mathbf{1 6}$ as a pale yellow amorphous product $(79.5 \mathrm{mg}, 79 \%)$. ${ }^{1} \mathrm{H}-\mathrm{NMR}$ (DMSO- $\left.d_{6}\right) \delta: 1.35(3 \mathrm{H}, \mathrm{s}$, $\left.\mathrm{CH}_{3}-2\right), 1.37\left(3 \mathrm{H}, \mathrm{s}, \mathrm{CH}_{3}-2\right), 2.05\left(3 \mathrm{H}, \mathrm{s}, \mathrm{OCOCH}_{3}\right), 2.07\left(3 \mathrm{H}, \mathrm{s}, \mathrm{OCOCH}_{3}\right)$, $2.30\left(3 \mathrm{H}, \mathrm{s}, \mathrm{CH}_{3}-5\right), 2.64\left(3 \mathrm{H}, \mathrm{s}, \mathrm{CH}_{3}-11\right), 3.84\left(3 \mathrm{H}, \mathrm{s}, \mathrm{OCH}_{3}\right), 5.16(1 \mathrm{H}, \mathrm{d}$, $J=5 \mathrm{~Hz}, \mathrm{C} 3-\mathrm{H}), 6.32(1 \mathrm{H}, \mathrm{d}, J=5 \mathrm{~Hz}, \mathrm{C} 4-\mathrm{H}), 7.05(1 \mathrm{H}, \mathrm{dd}, J=9,2 \mathrm{~Hz}, \mathrm{C} 8$ H), $7.38(1 \mathrm{H}, \mathrm{d}, J=9 \mathrm{~Hz}, \mathrm{C} 7-\mathrm{H}), 7.60(1 \mathrm{H}, \mathrm{d}, J=2 \mathrm{~Hz}, \mathrm{C} 10-\mathrm{H}), 10.80(1 \mathrm{H}$, $\mathrm{D}_{2} \mathrm{O}$ exch, s, NH). ${ }^{13} \mathrm{C}-\mathrm{NMR}\left(\mathrm{DMSO}-d_{6}\right) \delta: 12.7\left(\underline{\mathrm{CH}}_{3}-11\right), 13.3\left(\underline{\mathrm{CH}}_{3}-5\right)$, $20.9\left(\mathrm{OCOCH}_{3}\right), 21.1 \quad\left(\mathrm{OCOCH}_{3}\right), 22.1 \quad\left(\underline{\mathrm{CH}}_{3}-2\right), 26.4\left(\mathrm{CH}_{3}-2\right), 56.2$ $\left(\mathrm{OCH}_{3}\right), 64.6(\mathrm{C}-4), 72.2$ (C-3), 74.7 (C-2), 106.1 (C-10), 112.0 (C-7), 115.0 (C-8), 116.0 (C-4a), 116.6 (C-10b), 117.7 (C-5), 122.8 (C-11), 123.8 (C10a), 135.5 (C-5a), 136.6 (C-6a), 143.6 (C-11a), 153.1 (C-9), 170.2 $\left(\mathrm{COCH}_{3}\right), 170,6\left(\mathrm{COCH}_{3}\right) . \mathrm{IR} v_{\max } \mathrm{cm}^{-1}: 3424,2979,2936,2831,1734$, 1250, 1220, 1018. UV $\lambda_{\max } \mathrm{nm}(\log \varepsilon): 239.5$ (4.68), 250.5 (4.52), 262.5 (4.24), 273.5 (3.89), 298.5 (4.24), 308.5 (4.48). ESI-MS $m / z: 425$ $\left([\mathrm{M}+\mathrm{Na}]^{+}\right)$. Anal. Calcd for $\mathrm{C}_{24} \mathrm{H}_{27} \mathrm{NO}_{6}: \mathrm{C}, 67.75 ; \mathrm{H}, 6.40 ; \mathrm{N}, 3.29$. Found: $\mathrm{C}, 67.73 ; \mathrm{H}, 6.38 ; \mathrm{N}, 3.29$.

(3aRS,12bRS)-4,4,6,12-Tetramethyl-3a,4,11,12b-tetrahydro-1,3-dioxolo $\left[4^{\prime}, 5^{\prime}: 4,5\right]$ pyrano[3,2-b]carbazol-2-one (17) To a solution of 13 (103 $\mathrm{mg}, 0.33 \mathrm{mmol})$ in 2-butanone $(5 \mathrm{ml})$ was added $N, N^{\prime}$-carbonyldiimidazole (261 $\mathrm{mg}, 1.61 \mathrm{mmol})$. The reaction mixture was refluxed under Ar for $1.5 \mathrm{~h}$ and after cooling, aqueous $5 \% \mathrm{NaHCO}_{3}$ solution $(8 \mathrm{ml})$ was added. The solution was extracted with $\mathrm{CH}_{2} \mathrm{Cl}_{2}(3 \times 20 \mathrm{ml})$ and the combined organic layers were dried over $\mathrm{Na}_{2} \mathrm{SO}_{4}$, filtered, and evaporated under reduced pressure. Column chromatography of the crude product on silica gel (cyclohexane/ EtOAc: $5 / 5, \mathrm{v} / \mathrm{v})$ gave 17 as a whitish amorphous product $(48 \mathrm{mg}, 43 \%) .{ }^{1} \mathrm{H}-$ NMR (DMSO- $\left.d_{6}\right) \delta: 1.13\left(3 \mathrm{H}, \mathrm{s}, \mathrm{CH}_{3}-2\right), 1.51\left(3 \mathrm{H}, \mathrm{s}, \mathrm{CH}_{3}-2\right), 2.56(3 \mathrm{H}, \mathrm{s}$, $\left.\mathrm{CH}_{3}-5\right), 2.63\left(3 \mathrm{H}, \mathrm{s}, \mathrm{CH}_{3}-11\right), 5.07(1 \mathrm{H}, \mathrm{d}, J=8 \mathrm{~Hz}, \mathrm{C} 3-\mathrm{H}), 6.36(1 \mathrm{H}, \mathrm{d}$, $J=8 \mathrm{~Hz}, \mathrm{C} 4-\mathrm{H}), 7.13(1 \mathrm{H}, \mathrm{t}, J=8 \mathrm{~Hz}, \mathrm{C} 9-\mathrm{H}), 7.39(1 \mathrm{H}, \mathrm{t}, J=8 \mathrm{~Hz}, \mathrm{C} 8-\mathrm{H})$, $7.52(1 \mathrm{H}, \mathrm{d}, J=8 \mathrm{~Hz}, \mathrm{C} 7-\mathrm{H}), 8.13(1 \mathrm{H}, \mathrm{d}, J=8 \mathrm{~Hz}, \mathrm{C} 10-\mathrm{H}), 10.14\left(1 \mathrm{H}, \mathrm{D}_{2} \mathrm{O}\right.$ exch, s, NH). ${ }^{13} \mathrm{C}-\mathrm{NMR}$ (DMSO-d $\left.{ }_{6}\right) \delta: 12.6\left(\underline{\mathrm{CH}}_{3}-11\right), 13.5\left(\underline{\mathrm{CH}}_{3}-5\right), 22.4$ $\left(\underline{\mathrm{CH}}_{3}-2\right), 24.6\left(\underline{\mathrm{CH}}_{3}-2\right), 71.9(\mathrm{C}-4), 75.3(\mathrm{C}-2), 79.4(\mathrm{C}-3), 111.6(\mathrm{C}-7)$, 115.2 (C-4a), 117.8 (C-10b), 118.5 (C-5), 119.0 (C-9), 123.1 (C-10 and C11), 123.5 (C-10a), 126.2 (C-8), 134.9 (C-5a), 141.5 (C-6a), 143.7 (C-11a), $154.7(\mathrm{C}=\mathrm{O})$. IR $v_{\max } \mathrm{cm}^{-1}: 3365,3050,2979,2970,1782,1182,1006$, 744. UV $\lambda_{\max } \mathrm{nm}(\log \varepsilon): 241.5$ (4.56), 248.5 (4.51), 258 (4.23), 270 (3.84), 290.5 (3.99), 300.5 (4.28). ESI-MS m/z: $360\left([\mathrm{M}+\mathrm{Na}]^{+}\right)$. Anal. Calcd for $\mathrm{C}_{20} \mathrm{H}_{19} \mathrm{NO}_{4}$ : C, 71.20; H, 5.68; N, 4.15. Found: C, 71.16; H, 5.65; N, 4.16.

(3aRS,12bRS)-8-methoxy-4,4,6,12-tetramethyl-3a,4,11,12b-tetrahydro-1,3-dioxolo $\left[4^{\prime}, 5^{\prime}: 4,5\right]$ pyrano $[3,2-b]$ carbazol-2-one (18) Compound 18 was obtained from diol $14(100 \mathrm{mg}, 0.29 \mathrm{mmol})$ according to the same procedure as that described for the preparation of 17 , as a whitish amorphous product $(57 \mathrm{mg}, 53 \%) .{ }^{1} \mathrm{H}-\mathrm{NMR}$ (DMSO-d $) \delta: 1.28\left(3 \mathrm{H}, \mathrm{s}, \mathrm{CH}_{3}-2\right)$, $1.51\left(3 \mathrm{H}, \mathrm{s}, \mathrm{CH}_{3}-2\right), 2.53\left(3 \mathrm{H}, \mathrm{s}, \mathrm{CH}_{3}-5\right), 2.63\left(3 \mathrm{H}, \mathrm{s}, \mathrm{CH}_{3}-11\right), 3.84(3 \mathrm{H}, \mathrm{s}$, $\left.\mathrm{OCH}_{3}\right), 5.06(1 \mathrm{H}, \mathrm{d}, J=8 \mathrm{~Hz}, \mathrm{C} 3-\mathrm{H}), 6.35(1 \mathrm{H}, \mathrm{d}, J=8 \mathrm{~Hz}, \mathrm{C} 4-\mathrm{H}), 7.07(1 \mathrm{H}$, dd, $J=9,2 \mathrm{~Hz}, \mathrm{C} 8-\mathrm{H}), 7.42(1 \mathrm{H}, \mathrm{d}, J=9 \mathrm{~Hz}, \mathrm{C} 7-\mathrm{H}), 7.60(1 \mathrm{H}, \mathrm{d}, J=2 \mathrm{~Hz}$, $\mathrm{C} 10-\mathrm{H}), 10.93\left(1 \mathrm{H}, \mathrm{D}_{2} \mathrm{O}\right.$ exch, s, NH). ${ }^{13} \mathrm{C}-\mathrm{NMR}$ (DMSO-d $\left.{ }_{6}\right) \delta: 12.5\left(\mathrm{CH}_{3}-\right.$ 11), $13.4\left(\underline{\mathrm{CH}}_{3}-5\right), 22.5\left(\underline{\mathrm{CH}}_{3}-2\right), 24.6\left(\underline{\mathrm{CH}}_{3}-2\right), 56.2\left(\mathrm{O}_{\underline{C}} \mathrm{H}_{3}\right), 71.9(\underline{\mathrm{C}}-4)$, 75.3 (C-2), 79.4 (C-3), 106.0 (C-10), 112.2 (C-7), 115.2 (C-4a), 115.4 (C-8), 117.7 (C-10b), 118.6 (C-5), 122.9 (C-11), 123.8 (C-10a), 135.7 (C-5a), 136.5 (C-6a), 143.2 (C-11a), 153.2 (C-9), $154.7(\mathrm{C}=\mathrm{O})$. IR $v_{\max } \mathrm{cm}^{-1}$ : $3381,2982,2932,2823,1784,1225,1181$. UV $\lambda_{\max } n m(\log \varepsilon): 242.5$ (4.71), 251.5 (4.58), 263.5 (4.24), 301 (4.26), 309.5 (4.47). ESI-MS $\mathrm{m} / \mathrm{z}$ : $390\left([\mathrm{M}+\mathrm{Na}]^{+}\right)$. Anal. Calcd for $\mathrm{C}_{21} \mathrm{H}_{21} \mathrm{NO}_{5}: \mathrm{C}, 68.65 ; \mathrm{H}, 5.76 ; \mathrm{N}, 3.81$. Found: C, 68.64; H, 5.77; N, 3.81.

1,4,9-Trimethyl-9H-carbazol-3-ol (20) To an ice-cooled solution of 1,4,9-trimethyl- $9 H$-carbaldehyde $\left.{ }^{15}\right)(7.8 \mathrm{~g}, 35.0 \mathrm{mmol})$ in methanol $(400 \mathrm{ml})$ were added $33 \%$ aqueous hydrogen peroxide $(5 \mathrm{ml})$ and $95 \%$ sulfuric acid $(2.5 \mathrm{ml})$. After stirring for $15 \mathrm{~min}$ at $0{ }^{\circ} \mathrm{C}$, the reaction mixture was diluted with a mixture of crushed ice and water and neutralized with aqueous $1 \mathrm{M}$ $\mathrm{NaOH}$ solution to give a whitish precipitate which was filtered off and washed with water. Crystallization from $\mathrm{MeOH}$ gave 1,4,9-trimethyl- $9 \mathrm{H}$ carbazol-3-ol as white needles $(6.5 \mathrm{~g}, 88 \%)$, mp 208-209 ${ }^{\circ} \mathrm{C} .{ }^{1} \mathrm{H}-\mathrm{NMR}$ $\left(\mathrm{DMSO}-d_{6}\right) \delta: 2.61\left(3 \mathrm{H}, \mathrm{s}, \mathrm{CH}_{3}-1\right), 2.72\left(3 \mathrm{H}, \mathrm{s}, \mathrm{CH}_{3}-4\right), 4.00\left(3 \mathrm{H}, \mathrm{s}, \mathrm{NCH}_{3}\right)$, $6.79(1 \mathrm{H}, \mathrm{s}, \mathrm{C} 2-\mathrm{H}), 7.12(1 \mathrm{H}, \mathrm{td}, J=8,1 \mathrm{~Hz}, \mathrm{C} 6-\mathrm{H}), 7.39(1 \mathrm{H}, \mathrm{td}, J=8,1 \mathrm{~Hz}$, C7-H), $7.48(1 \mathrm{H}, \mathrm{dd}, J=8,1 \mathrm{~Hz}, \mathrm{C} 8-\mathrm{H}), 8.11(1 \mathrm{H}, \mathrm{dd}, J=8,1 \mathrm{~Hz}, \mathrm{C} 5-\mathrm{H})$, $8.73\left(1 \mathrm{H}, \mathrm{D}_{2} \mathrm{O}\right.$ exch, s, OH). ${ }^{13} \mathrm{C}-\mathrm{NMR}$ (DMSO- $\left.d_{6}\right) \delta: 13.5\left(\mathrm{CH}_{3}-1\right), 20.8$ $\left(\mathrm{CH}_{3}-4\right), 33.1\left(\mathrm{NCH}_{3}\right), 109.8(\mathrm{C}-8), 115.5(\mathrm{C}-4 \mathrm{a}), 118.8(\mathrm{C}-4), 119.0(2 \mathrm{C}$, C-2 and C-6), 123.2 (C-5), 123.5 (C-1), 124.1 (C-4b), 125.8 (C-7), 134.5 (C-9a), 142.9 (C-8a), 148.6 (C-3). IR $v_{\max } \mathrm{cm}^{-1}:$ 3445, 1473, 1394, 1299, 750. UV $\lambda_{\max } \mathrm{nm}(\varepsilon): 227$ (4.17), 243.5 (4.26), 255 (3.97), 272 (3.90), 295.5 (3.81). CI-MS $m / z$ : $226\left([\mathrm{MH}]^{+}\right)$. Anal. Calcd for $\mathrm{C}_{15} \mathrm{H}_{15} \mathrm{NO}$ : C, 79.97; H, $6.71 ; \mathrm{N}, 6.22$. Found: C, 79.97; H, 6.72; N, 6.22.

2,2,5,6,11-Pentamethyl-2,6-dihydropyrano[3,2-b]carbazole (19) So- dium hydride ( $50 \%$ dispersion in oil, $160 \mathrm{mg}, 6.66 \mathrm{mmol})$ was added to a stirred solution of $20(750 \mathrm{mg}, 3.33 \mathrm{mmol})$ in dry toluene $(20 \mathrm{ml})$ under Ar. 3-Chloro-3-methylbut-1-yne ${ }^{20)}(1.70 \mathrm{~g}, 16.6 \mathrm{mmol})$ and potassium iodide $(2.75 \mathrm{~g}, 16.6 \mathrm{mmol})$ were then added. The reaction mixture was refluxed for $17 \mathrm{~h}$, cooled, washed with water, dried over $\mathrm{Na}_{2} \mathrm{SO}_{4}$, filtered, and evaporated under reduced pressure. Column chromatography of the crude product on silica gel (cyclohexane/ $\mathrm{CH}_{2} \mathrm{Cl}_{2}: 6 / 4, \mathrm{v} / \mathrm{v}$ ) gave 19 which crystallized from the eluent as pale yellow needles $(243 \mathrm{mg}, 25 \%)$, mp 180-181 ${ }^{\circ} \mathrm{C} .{ }^{1} \mathrm{H}-\mathrm{NMR}$ $\left(\mathrm{CDCl}_{3}\right) \delta: 1.48\left(6 \mathrm{H}, \mathrm{s},\left(\mathrm{CH}_{3}\right)_{2}-2\right), 2.74\left(3 \mathrm{H}, \mathrm{s}, \mathrm{CH}_{3}-5\right), 2.78\left(3 \mathrm{H}, \mathrm{s}, \mathrm{CH}_{3}-\right.$ 11), $4.08\left(3 \mathrm{H}, \mathrm{s}, \mathrm{NCH}_{3}\right), 5.82(1 \mathrm{H}, \mathrm{d}, J=12 \mathrm{~Hz}, \mathrm{C} 3-\mathrm{H}), 6.81(1 \mathrm{H}, \mathrm{d}$, $J=12 \mathrm{~Hz}, \mathrm{C} 4-\mathrm{H}), 7.18(1 \mathrm{H}, \mathrm{dd}, J=8,7 \mathrm{~Hz}, \mathrm{C} 9-\mathrm{H}), 7.35(1 \mathrm{H}, \mathrm{d}, J=8 \mathrm{~Hz}, \mathrm{C} 7-$ $\mathrm{H}), 7.44(1 \mathrm{H}, \mathrm{dd}, J=8,7 \mathrm{~Hz}, \mathrm{C} 8-\mathrm{H}), 8.20(1 \mathrm{H}, \mathrm{d}, J=8 \mathrm{~Hz}, \mathrm{C} 10-\mathrm{H}) .{ }^{13} \mathrm{C}-$ NMR $\left(\mathrm{CDCl}_{3}\right) \delta: 12.2\left(\underline{\mathrm{CH}}_{3}-5\right), 14.3\left(\mathrm{CH}_{3}-11\right), 27.1\left(2 \mathrm{C},\left(\underline{\mathrm{CH}}_{3}\right)_{2}-2\right), 33.4$ $\left(\mathrm{NCH}_{3}\right), 74.1(\mathrm{C}-2), 108.4(2 \mathrm{C}, \mathrm{C}-7$ and $\mathrm{C}-4 \mathrm{a}), 113.2$ (C-10b), $117.4(\mathrm{C}-5)$, 118.4 (C-9), 119.6 (C-11), 120.6 (C-10), 122.5 (C-4), 123.9 (C-10a), 124.9 (C-8), 131.8 (C-3), 135.5 (C-5a), 143.2 (C-6a), 144.1 (C-11a). IR $v_{\max } \mathrm{cm}^{-1}$ : $3433,3048,2968,2920,2862,1467,1300,1133,745,715$. UV $\lambda_{\max } \mathrm{nm}$ ( $\log \varepsilon$ ): 260.5 (4.53), 302 (3.94), 314 (4.77), 326.5 (4.37). CI-MS m/z: 292 $\left([\mathrm{MH}]^{+}\right)$. Anal. Calcd for $\mathrm{C}_{20} \mathrm{H}_{21} \mathrm{NO}$ : C, 82.44; H, 7.26; N, 4.81. Found: C, 82.50; H, 7.27; N, 4.82.

9-Methoxy-2,2,5,6,11-pentamethyl-2,6-dihydropyrano[3,2-b]carbazole (22) Sodium hydride ( $50 \%$ dispersion in oil, $10 \mathrm{mg}, 0.4 \mathrm{mmol}$ ) was added to an ice-cooled solution of $12(25.0 \mathrm{mg}, 0.08 \mathrm{mmol})$ in dry $N, N$-dimethylformamide $(5 \mathrm{ml})$. After stirring for $10 \mathrm{~min}$ at $0^{\circ} \mathrm{C}$, methyl iodide $(17.0 \mathrm{mg}$, $0.12 \mathrm{mmol}$ ) was added and the reaction mixture was stirred for $5 \mathrm{~min}$ at $0{ }^{\circ} \mathrm{C}$. The reaction mixture was diluted with ice water, extracted with $\mathrm{CH}_{2} \mathrm{Cl}_{2}$ $(3 \times 10 \mathrm{ml})$, and the combined organic layers were dried over $\mathrm{Na}_{2} \mathrm{SO}_{4}$, filtered, and evaporated under reduced pressure. Column chromatography of the crude product on silica gel (cyclohexane/EtOAc: 99/1, v/v) gave 22 which crystallized from the eluent as pale yellow needles $(23 \mathrm{mg}, 89 \%), \mathrm{mp}$ $166-167^{\circ} \mathrm{C} .{ }^{1} \mathrm{H}-\mathrm{NMR}\left(\mathrm{CDCl}_{3}\right) \delta: 1.48\left(6 \mathrm{H}, \mathrm{s},\left(\mathrm{CH}_{3}\right)_{2}-2\right), 2.70\left(3 \mathrm{H}, \mathrm{s}, \mathrm{CH}_{3}-\right.$ 5), $2.75\left(3 \mathrm{H}, \mathrm{s}, \mathrm{CH}_{3}-11\right), 3.94\left(3 \mathrm{H}, \mathrm{s}, \mathrm{OCH}_{3}\right), 4.02\left(3 \mathrm{H}, \mathrm{s}, \mathrm{NCH}_{3}\right), 5.82(1 \mathrm{H}$, d, $J=10 \mathrm{~Hz}, \mathrm{C} 3-\mathrm{H}), 6.79(1 \mathrm{H}, \mathrm{d}, J=10 \mathrm{~Hz}, \mathrm{C} 4-\mathrm{H}), 7.08(1 \mathrm{H}, \mathrm{dd}, J=9$, $2.5 \mathrm{~Hz}, \mathrm{C} 8-\mathrm{H}), 7.24(1 \mathrm{H}, \mathrm{d}, J=9 \mathrm{~Hz}, \mathrm{C} 7-\mathrm{H}), 7.71(1 \mathrm{H}, \mathrm{d}, J=2.5 \mathrm{~Hz}, \mathrm{C} 10-\mathrm{H})$. ${ }^{13} \mathrm{C}-\mathrm{NMR}\left(\mathrm{CDCl}_{3}\right) \delta$ : $12.1\left(\underline{\mathrm{CH}}_{3}-11\right), 14.3\left(\underline{\mathrm{CH}}_{3}-5\right), 27.1\left(2 \mathrm{C},\left(\underline{\mathrm{CH}}_{3}\right)_{2}-2\right)$, $33.7\left(\mathrm{NCH}_{3}\right), 56.0\left(\mathrm{OCH}_{3}\right), 74.1(\mathrm{C}-2), 106.4(\mathrm{C}-10), 108.9(\mathrm{C}-7), 113.3(\mathrm{C}-$ 8), 113.5 (C-4a), 117.2 (C-10b), 119.8 (C-5), 120.5 (C-4), 122.5 (C-11) 124.2 (C-10a), 128.8 (C-5a), 130.8 (C-6a), 131.9 (C-3), 144.4 (C-11a), 153.0 (C-9). IR $v_{\max } \mathrm{cm}^{-1}: 3448,3045,2967,2932,2827,1484,1222,1156$. UV $\lambda_{\max } \mathrm{nm}(\varepsilon): 259.5$ (4.53), 322 (4.33), 334.5 (4.44). CI-MS $m / z: 322$ $\left([\mathrm{MH}]^{+}\right)$. Anal. Calcd for $\mathrm{C}_{21} \mathrm{H}_{23} \mathrm{NO}_{2}: \mathrm{C}, 78.47 ; \mathrm{H}, 7.21 ; \mathrm{N}, 4.36$. Found: C, 78.52; H, 7.22; N, 4.35.

Cytotoxicity Murine leukaemia L1210 cells from the American Type Culture Collection (Rockville Pike, MD) were grown in RPMI medium 1640 supplemented with $10 \%$ fetal calf serum, $2 \mathrm{mM} \mathrm{L-glutamine,} 100 \mathrm{U} / \mathrm{ml}$ penicillin, $100 \mu \mathrm{g} / \mathrm{ml}$ streptomycin and $10 \mathrm{~mm}$ HEPES buffer (pH 7.4). The cytotoxicity was measured by microculture tetrazolium assay essentially as described. ${ }^{23}$ Cells were exposed for $48 \mathrm{~h}$ to nine graded concentrations in triplicate of the test drug. Results are expressed as $\mathrm{IC}_{50}$ (mean, $n=3$ ), which is defined as the drug concentration inhibiting the absorbance by $50 \%$ with respect to that of untreated cells.

\section{References}

1) Knölker H.-J., Reddy K. R., Chem. Rev., 102, 4303 - 4427 (2002).

2) Gribble G. W., "The Alkaloids: Chemistry and Pharmacology," Vol. 39, ed. by Brossi A., Academic Press, San Diego, 1990, pp. 239-352.

3) Mathé G., Hayat M., de Vassal F., Schwartzenberg L., Schneider M., Schlumberger J. R., Jasmin C., Rosenfeld C., Rev. Eur. Etud. Clin. Biol., 15, 541-545 (1970).

4) Bush J. A., Long B. H., Catino J. J., Bradner W. T., Tomita K., J. Antibiot., 40, 668-678 (1987).

5) Prudhomme M., Eur. J. Med. Chem., 38, 123-140 (2003).

6) Hughes G. K., Lahey F. N., Price J. R., Webb L. J., Nature (London), 162, 223-224 (1948).

7) Svoboda G. H., Lloydia, 29, 206-224 (1966).

8) Tillequin F., Michel S., Skaltsounis A.-L., "Alkaloids: Chemical and Biological Perspectives," Vol. 12, ed. by Pelletier S. W., Elsevier, New York, 1998, pp. 1-102.

9) Brum-Bousquet M., Mitaku S., Skaltsounis A.-L., Tillequin F., Koch M., Planta Med., 54, 470-471 (1988).

10) Elomri A., Mitaku S., Michel S., Skaltsounis A.-L., Tillequin F., Koch M., Pierré A., Guilbaud N., Léonce S., Kraus-Berthier L., Rolland Y., Atassi Gh., J. Med. Chem., 39, 4762-4766 (1996).

11) Costes N., Le Deit H., Michel S., Tillequin F., Koch M., Pfeiffer B., 
Renard P., Léonce S., Guilbaud N., Kraus-Berthier L., Pierré A., Atassi Gh., J. Med. Chem., 43, 2395-2402 (2000).

12) Guilbaud N., Léonce S., Tillequin F., Koch M., Hickman J. A., Pierré A., Anti-Cancer Drugs, 13, 445-449 (2002).

13) David-Cordonnier M.-H., Laine W., Lansiaux A., Kouach M., Briand G., Pierré A., Hickman J. A., Bailly C., Biochemistry, 41, 9911—9920 (2002).

14) Doan-Thi-Mai H., Gaslonde T., Michel S., Tillequin F., Koch M., Bongui J.-B., Elomri A., Seguin E., Pfeiffer B., Renard P., David-Cordonnier M.-H., Laine W., Bailly C., Kraus-Berthier L., Léonce S., Hickman J. A., Pierré A., J. Med. Chem., 46, 3072-3082 (2003).

15) Dalton L. K., Demerac S., Elmes B. C., Loder J. W., Swan J. M., Teitei T., Aust. J. Chem., 20, 2715-2727 (1967).

16) Langendoen A., Koomen G.-J., Pandit U. K., Heterocycles, 26, 91-94 (1987).
17) Chauder B. A., Lopes C. C., Lopes R. S., da Silva A. J. M., Snieckus V., Synthesis, 1998, 279-282 (1998).

18) Van Rheenen V., Kelly R. C., Cha D. Y., Tetrahedron Lett., 23, 1973 1976 (1976).

19) Evans J. M., Fake C. S., Hamilton T. C., Poyser R. H., Watts E. A., J. Med. Chem., 26, 1582-1589 (1983).

20) Hennion G. F., Boisselle A. P., J. Org. Chem., 26, 725-727 (1961).

21) Furukawa H., Yogo M., Ito C., Wu T.-S., Kuoh C.-S., Chem. Pharm. Bull., 33, 1320-1322 (1985).

22) Kumar V., Reisch J., Wickramasinghe A., Aust. J. Chem., 42, 13751379 (1989).

23) Pierré A., Kraus-Berthier L., Atassi Gh., Cros S., Poupon M. F., Lavielle G., Berlion M., Bizarri J. P., Cancer Res., 51, 2312-2318 (1991). 